\title{
Diagnosis of depression by MRI scans with the use of VSRAD - a promising auxiliary means of diagnosis: a report of 10 years research
}

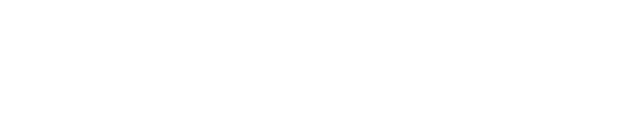

Richi Niida'

Akira $\mathrm{Niida}^{2}$

Makoto Motomura ${ }^{3}$

Akihiko Uechi ${ }^{4}$

'Nanto Clinic, Urasoe City, Okinawa, Japan; ${ }^{2}$ Nanbu Hospital, Itoman City, Okinawa, Japan; ${ }^{3}$ University of the Ryukyus, Nakagami County, Okinawa, Japan; ${ }^{4}$ Kansai Gaidai University, Hirakata City, Osaka, Japan
Correspondence: Richi Niida Nanto Clinic 3-3-7 Gusukuma, Urasoe City, Okinawa, 90I-2133, Japan Tel +8I-98-988-3208

Fax +8I-98-988-3207

Email rniida@nirai.ne.jp
Objectives: This study was designed to evaluate the usefulness of assessing subgenual anterior cingulate cortex (sACC) volume reduction by magnetic resonance imaging (MRI) as an objective auxiliary means of diagnosis of depression. The study was additionally designed to analyze the association of sACC volume reduction with the effectiveness of treatments for depression and other diseases presenting with similar symptoms, and to examine the possibility of using sACC volume reduction in the distinction between depression and bipolar disorder and determining optimum medication for these conditions.

Methods: Three-dimensional T1-weighted sagittal images, taken with Achieva 1.5T NOVA (Philips), were analyzed with VSRAD plus ${ }^{\circledR}$ to evaluate a reduction in sACC volume. The finding from this analysis was compared with the clinical data, including the longitudinal course follow-up data based on the treatment algorithm.

Subjects: The study involved 88 patients aged over 54 who received MRI during 2010, ie, 71 patients with major depressive disorder (MDD), 11 patients bipolar disorder, and 6 patients in whom the initial diagnosis (MDD) was later modified. Thirty-three normal individuals served as controls.

Results: sACC volume reduction was noted in 66 of the 71 patients receiving treatment of MDD, with sensitivity of $93 \%$, specificity of $85 \%$, and accuracy of $90 \%$. In the 66 patients diagnosed as having MDD and exhibiting SACC volume reduction, the disease showed remission in response to treatment with antidepressants, but medication needed to be continued after achievement of remission. In cases initially diagnosed as having MDD but not exhibiting sACC volume reduction, the necessity of modifying the diagnosis was considered. Typical cases of bipolar disorder did not exhibit sACC volume reduction.

Conclusion: That patients receiving treatment of MDD often showed reduction in SACC volume suggests the usefulness of this parameter as an objective auxiliary means of diagnosis for MDD.

Keywords: depression, subgenual anterior cingulate cortex (sACC), MRI, VSRAD, neuroimaging

\section{Introduction}

Depression is a disease difficult to diagnose, because it needs to be distinguished from various other diseases for the following reasons: 1) depression is sometimes accompanied by seasonal affective disorder, obsessive-compulsive disorder, or posttraumatic stress disorder; 2) depression sometimes presents with symptoms similar to early symptoms of schizophrenia; and 3) depression occasionally appears at the early stage of dementia in elderly people. Failure to diagnose and treat depression 
appropriately can result in unfortunate outcomes, leading to suicide in worst cases. Dropout from medical treatment is also seen among patients with depression, for reasons such as adverse reactions at early stages of antidepressant therapy or failure to establish a satisfactory doctor-patient relationship. The absence of a reliable test assisting the diagnosis and treatment of depression is considered to be one of the major factors responsible for failure in establishment of a satisfactory doctor-patient relationship. At present, the diagnosis of depression is based on assessing the patient's experience and behavior by means of interview and comparing the interview findings with diagnostic criteria such as the Diagnostic and Statistical Manual of Mental Disorders-IV (DSM-IV). DSM-IV classification of psychiatric disorders is based on symptomatological findings, rather than on the pathophysiology or the mechanism for pathogenesis. In other words, depression is now diagnosed as a group of disorders which in nature involve varying features. For this reason, depression diagnosed in this way is composed of a mixture of diverse features, making it difficult to identify a feature specific to depression.

Chronological evaluation of the efficacy used for treatment of depression and the choice of drugs for treatment of this disease also face similar limitations. Objective data such as biological indicators measured by laboratory tests are never used for such purposes. Lack of an established biological indicator of depression tends to reduce the motivation of patients towards treatment and to make it difficult for physicians to have common recognition about treatment of this disease, thus serving as one of the factors responsible for interruption of treatment.

Depression has conventionally been viewed as repeating cycles of multiple phases and showing alleviation towards normal condition in response to treatment. In clinical practice, however, there are cases of depression where treatment with antidepressants and sleeping pills cannot be completed in much time even after alleviation of the disease phase or cases where cognitive function does not return to the predisease level. Many depressed patients with poor prognosis respond poorly to both drug therapy and nondrug therapy (eg, cognitive behavioral therapy), which makes physicians feel limited in their management despite attempts to take adequate measures. How to deal with these cases is a difficult issue for physicians involved in clinical care of depression.

In recent years, the strategy for diagnosis of psychiatric diseases has been undergoing modifications following advances in diagnostic imaging and molecular biology. In cases of bipolar disorder, involvement of mitochondrial dysfunction and stress-induced reactions of endoplasmic reticulum has been reported. Patients with depression have been reported to show changes (often decrease) in grey matter volume of the anterior cingulated cortex, amygdala, hippocampus, and prefrontal area. Pezawas et $\mathrm{al}^{1}$ reported that the presence of S-type serotonin transporter gene promoter affects intrauterine brain development, causing volume reduction of the subgenual anterior cingulated cortex (sACC). Since that report, the association between the brain area sACC and depression has been attracting close attention.

This paper describes our attempt at longitudinal and crosssectional research of the volume-reduced brain areas in patients with depression through whole brain analysis using a magnetic resonance imaging (MRI) system equipped with a Voxel-based Specific Regional analysis system for Alzheimer's Disease (VSRAD plus ${ }^{\circledR}$ ), a freeware computer program developed by Matsuda et al, ${ }^{2}$ as a means of diagnosing disorders associated with early Alzheimer dementia. The authors show that patients receiving treatment of major depressive disorder (MDD) have volume reduction more frequently in SACC than in the other regions (amygdala, hippocampus, and prefrontal area) and propose that checking for sACC volume reduction is useful as an objective auxiliary means of diagnosing MDD.

\section{Objectives}

The objectives of this study are 3-fold. Firstly, we evaluate the usefulness of VSRAD as an objective auxiliary means of diagnosing depression through analyzing the clinical data of depressed patients over 10 years with the findings of sACC atrophy (sACC volume reduction) as measured with MRI VSRAD. Secondly, we evaluate the association of the presence/absence of sACC volume reduction with the effectiveness of medication for depression or diseases presenting with similar symptoms through analysis of the longitudinal course of treatment based on a drug treatment algorithm. Thirdly, we examine the possibility of utilizing sACC volume reduction data to distinguish between depression and bipolar disorder (often needed during clinical practice) and in selection of optimum medication for these diseases.

\section{Subjects}

During the 6-year period from 2001 to 2006, the authors accepted and managed 2652 patients as inpatients, including those referred from psychiatrists working in other medical facilities in Okinawa Prefecture, providing inpatient care in parallel with care for other patients. During the subsequent 
4-year period (2007-2010), the authors provided only outpatient care. Of the patients managed during this 10-year period, those able to receive MRI during 2010 and aged over 54 (because the database for healthy individuals in VSRAD covered individuals aged between 54 and 86) were adopted as subjects of this study. (This study was made with adequate consideration to keep anonymity of individual patients and to avoid disclosure of personal information. Consent to this report has been obtained from all patients involved).

In total, 88 patients were enrolled to this study, including 71 patients diagnosed as having MDD according to the DSM-IV diagnostic criteria, aged over 54 and receiving antidepressant therapy, 11 patients diagnosed as having bipolar disorder according to the DSM-IV diagnostic criteria, and 6 patients in whom the initial diagnosis (MDD) was later modified. In view of the features of VARAD which covers elderly individuals as well, the following patients were excluded from this study: 1) patients in whom dementia developed after onset of depression, 2) patients in whom the management period was not long enough to allow definite diagnosis, and 3) patients unable to be followed at our facility due to a change to other medical facilities.

The 71 patients with MDD (12 males and 59 females) were aged between 54 and 82 years (mean: $65.4 \pm 6.5$ ). Complication by anxiety disorder or panic disorder was seen in $31(44 \%)$ of these 71 patients with MDD. The 11 patients with bipolar disorder ( 2 males and 9 females) were aged between 54 and 72 (mean $62.7 \pm 4.8$ ). The 6 patients in whom the initial diagnosis was later modified ( 5 males and 1 female) were aged between 55 and 76 years (mean $66.5 \pm 5.2$ ). For comparison with the MDD group, a healthy control group was examined in the study. The healthy control group was composed of 33 individuals who had no history of MDD and received MRI to rule out organic disease suspected for some reason ( 7 males and 26 females aged between 54 and 84 years, with a mean age of $63.3 \pm 5.7$ ) (Table 1 ).

Evaluation of the 71 patients with MDD on the basis of the drug treatment algorithm yielded the following results. The duration of illness was 8 to 12 months on average. The treatment period ranged from 5 months to 10 years. HAM-D (Hamilton's Rating Scale for Depression) value ranged from 10 to 30 (mean $17.8 \pm 3.0$ ). Age at onset of MDD was $61.2 \pm 6.9$ years. Age at the time of evaluation was 54 to 63 years in 33 cases, 64 to 73 years in 25 cases, and $>74$ years in 13 cases. When the duration of illness was divided into 5 categories, it was $<6$ months in none, $>6$ months, and $<1$ year in 9 cases, $>1$ year and $<3$ years in 24 cases, $>3$ years and $<6$ years in 19 cases and $>6$ years in 19 cases. When the treatment period was divided into 5 categories, it was $<6$ months in 6 cases, $>6$ months and $<1$ year in 15 cases, $>1$ year and $<3$ years in 20 cases, $>3$ years and $<6$ years in 21 cases, and $>6$ years in 9 cases. The disease severity score according to HAM-D classification was $<13$ in 7 cases, 14 to 18 in 39 cases, 19 to 22 in 15 cases, and $>23$ in 10 cases. The drug prescribed at the time of MRI was selective serotonin reuptake inhibitors (SSRI) in 61 cases, serotonin and norepinephrine reuptake inhibitors (SNRI) in 9 cases, noradrenergic and specific serotonergic antidepressants (mirtazapine) in 5 cases, sulpiride in 3 cases, tricyclic antidepressant in 2 cases, and tetracyclic antidepressant in 1 case (including some cases receiving 2 or more drugs at a time).

For $25(35 \%)$ of the 71 patients with MDD, the prescribed drug was at the maintenance dose level (the antidepressant dose level kept at a certain level for 1 year or longer) at the time of MRI. The disease was in the phase of remission (HAM-D score $<7)$ in $35(49 \%)$ of the 71 patients at the time of MRI. Thus, half of all patients with MDD received MRI when the disease had alleviated.

\section{Methods}

VSRAD used in the present study to assess volume reduction in local brain grey matter is a free computer program designed to evaluate relative local brain volume of individual patients through comparison of visual information from MRI with the brain image database for healthy individuals by means of voxelbased morphometry (VBM). With this program, the 3D-T1 weighted image of the entire brain, taken with a 1.5-Tesla MRI device, is processed with SPM2 (Statistical Parametric Mapping, 2002 Edition) to isolate grey matter, followed by automated statistical analysis of grey matter density in units of voxel for the entire brain based on anatomical standardization (not involving any hypothesis). For statistical analysis in comparison to the image database for healthy individuals, $Z$ score (the magnitude of grey matter density's discrepancy, $\mathrm{n} \times$ standard deviation (SD), in individual patients from the mean for healthy individuals) is calculated for each brain region, using the average image and SD contained in the image database. The results of analysis are displayed as a colored scale map on the standard brain. The database for healthy individuals includes 40 Japanese males and 40 Japanese females aged between 54 and 86 years $(70.2 \pm 7.3)$.

In the present study, 3-dimensional T1-weighted sagittal images were taken with an Achieve 1.5T NOVA (Philips) under the following setting: FOV 240, matrix $256 \times 256$, 
Table I Clinical characteristics of the total sample $(n=12 I)$

\begin{tabular}{|c|c|c|c|c|c|c|c|c|c|}
\hline \multirow[t]{2}{*}{ Characteristics } & \multicolumn{4}{|l|}{ Mean (SD) } & \multirow[t]{2}{*}{$H$} & \multirow[t]{2}{*}{$U$} & \multirow[t]{2}{*}{$\chi^{2}$} & \multirow[t]{2}{*}{ df } & \multirow[t]{2}{*}{$P$ value } \\
\hline & MDD & BD & DC & $\mathrm{HC}$ & & & & & \\
\hline Sample, no. & 71 & 11 & 6 & 33 & & & & & \\
\hline \multicolumn{10}{|l|}{ Sex, no. } \\
\hline Male & 12 & 2 & 5 & 7 & & & 14.56 & 3 & 0.002 \\
\hline Female & 59 & 9 & I & 26 & & & & & \\
\hline Age, y & $65.37(6.55)$ & $62.73(4.79)$ & $66.50(5.17)$ & $63.36(5.69)$ & 2.95 & & & 3 & 0.4 \\
\hline Complication $^{\mathrm{a}}$ & 31 & & & & & & & & \\
\hline HAMD, point & I7.8(3.0) & & $17.5(1.33)$ & & & 214.5 & & & 0.49 \\
\hline-13 & 7 & & 0 & & & & & & \\
\hline $14-18$ & 39 & & 4 & & & & & & \\
\hline $19-22$ & 15 & & 2 & & & & & & \\
\hline $23-$ & 10 & & 0 & & & & & & \\
\hline Age at onset, $y$ & $61.20(6.86)$ & $55.46(5.32)$ & $61.5(5.67)$ & & 5.04 & & & 2 & 0.08 \\
\hline-53 & 0 & 5 & I & & & & & & \\
\hline $54-63$ & 33 & 5 & I & & & & & & \\
\hline $64-73$ & 25 & 1 & 4 & & & & & & \\
\hline $74-$ & 13 & 0 & 0 & & & & & & \\
\hline Duration of illness, $y$ & $4.4 \mathrm{I}(2.34)$ & $7.27(1.70)$ & $5.33(3.11)$ & & 9.22 & & & 2 & 0.01 \\
\hline-0.5 & 0 & 0 & 0 & & & & & & \\
\hline $0.5-1$ & 9 & 0 & 0 & & & & & & \\
\hline $\mathrm{I}-3$ & 24 & 0 & I & & & & & & \\
\hline $3-6$ & 19 & 2 & 3 & & & & & & \\
\hline $6-$ & 19 & 9 & 2 & & & & & & \\
\hline Duration of treatment, $y$ & $2.80(1.99)$ & $7.27(1.70)$ & $4.23(2.5 I)$ & & 20.43 & & & 2 & $P<0.001$ \\
\hline-0.5 & 6 & 0 & 0 & & & & & & \\
\hline $0.5-1$ & 15 & 0 & I & & & & & & \\
\hline $\mathrm{I}-3$ & 20 & 0 & 2 & & & & & & \\
\hline $3-6$ & 21 & 2 & I & & & & & & \\
\hline $6-$ & 9 & 9 & 2 & & & & & & \\
\hline \multicolumn{10}{|c|}{ Drug use (include combined use) } \\
\hline SSRI & 61 & & & & & & & & \\
\hline SNRI & 9 & & & & & & & & \\
\hline NaSSA & 5 & & & & & & & & \\
\hline Sulpiride & 3 & & & & & & & & \\
\hline Tricycle anti-dep & 2 & & & & & & & & \\
\hline Tetracycle ant-dep & 1 & & & & & & & & \\
\hline
\end{tabular}

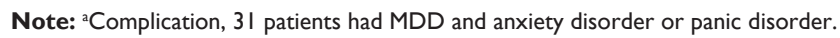

Abbreviations: anti-dep, antidepressant; BD, bipolar disorder; DC, diagnostic change case; H, Kruskal-Wallis nonparametric multiple sample test; HAMD, Hamilton's Rating Scale for Depression; HC, healthy controls; MDD, major depression disorder; NaSSA, noradrenergic and specific serotonergic antidepressant; SNRI, serotonin noradrenaline reuptake inhibitor; SSRI, selective serotonin reuptake inhibitor; $U$, Mann-Whitney nonparametric 2-sample test.

slice thickness $1 \mathrm{~mm}, 160$ slices, TR $9.3 \mathrm{msec}$, TE $4.6 \mathrm{msec}$, flip angle 10 degrees. The obtained visual data were analyzed on a PC, with the program VSRAD plus ${ }^{\circledR}$. The $Z$ score map obtained was checked for the presence/absence of colored area, which indicates volume reduction (attenuation of signal density in the grey matter) within the regions of interest. On the brain image thus obtained, the areas showing volume reduction by $Z$ score $\geq 2$ or more are designed to be colored blue. We visually checked the presence/absence of sACC volume reduction on the images with ROI (region of interest) set in the sACC. The finding as to presence/absence of volume reduction in sACC, hippocampus, amygdale, and prefrontal area of individual patients was compared with the clinical data from the same patients for the previous 10-year period. Chi-square for independence test was employed for statistical analysis.

\section{Results}

\section{Association between MDD and sACC volume reduction (Figure I)}

Of the 71 patients with MDD, 66 showed sACC volume reduction. Volume reduction in hippocampus, prefrontal area, and amygdala was noted in 35, 20, and 21 patients, respectively. sACC volume reduction as an indicator for the diagnosis of MDD had a sensitivity of $93 \%$, specificity of $85 \%$, and accuracy of $90 \%$. In the MDD group, sACC volume 


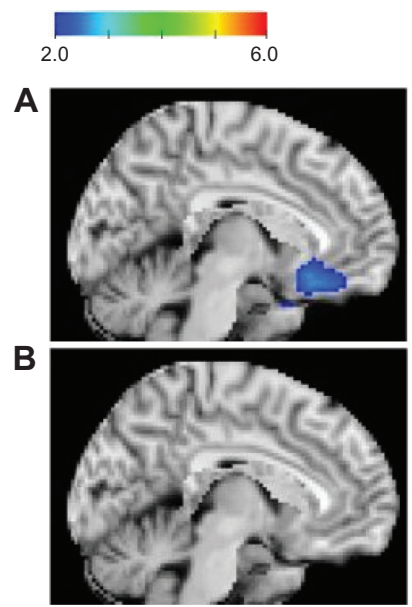

Left medial

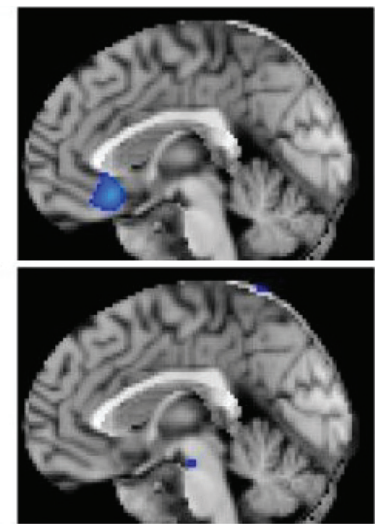

Right medial
Figure I A) Z-score maps of a 58-year-old woman with major depressive disorder (MDD). B) A 60-year-old normal woman (control). The color scale for Z score is shown in the upper left of the figure. Presented in color if $Z$ score is $>2$. In the patient with MDD (A) the subgenual cingulate gyrus and the surrounding region are colored blue, indicating volume reduction. In the normal control (B), no colored region, reflecting volume reduction, is visible.

reduction was noted more frequently than volume reduction in any other region of the brain (Table 2).

Of the 11 patients with bipolar disorder, 6 showed sACC volume reduction. sACC volume reduction was not seen in any of the 6 patients in whom the initial diagnosis was later modified. Of the 33 normal controls, 5 showed sACC volume reduction.

The MDD group showed significant sACC volume reduction measured by MRI compared with the normal control group, regardless of gender, age, duration of illness, treatment period, length of time before remission, disease severity, accompanying physical disease, type of antidepressant used, drug dose level, or dosing period (Table 3). Of the 71 patients with MDD, 35 showed volume reduction in the hippocampus, 20 showed volume reduction in the prefrontal area, and 21 showed volume reduction in the amygdale.

\section{Association between sACC volume reduction and diagnosis/treatment of MDD}

For the course of treatment and its efficacy, the patients who received drug therapy after diagnosis of MDD in accordance with DSM-IV diagnostic criteria could be divided into the following 2 groups depending on the presence/absence of sACC volume reduction, and each group had different characteristics:

1. Group showing sACC volume reduction (66/71 cases)

2. Group without sACC volume reduction (5/71 cases)

The characteristic findings from each group can be summarized as:

1. Group showing sACC volume reduction (66 cases): A characteristic of the course of treatment in this group was favorable responses to antidepressant treatment such as SNRI or SSRI seen in many cases. In this group, the length of time until remission of symptoms ( $50 \%$ or more decrease in HAMD score) following the start of antidepressant treatment was short and was 3 months in 43 $(65 \%)$ of the 66 cases. The disease alleviated rapidly after antidepressants inappropriate for individual cases were determined. However, all cases required maintenance antidepressant therapy even after remission. Discontinuation of antidepressant intake at the patient's own discretion was seen in 15 cases. In all of these 15 cases, symptoms relapsed. Thus, in cases showing sACC volume reduction, treatment with antidepressants resulted in remission of the disease, but continuation of drug therapy was needed after remission.

2. Group without sACC volume reduction (5/71 cases): In this group, all patients treated with antidepressants showed remission of the disease within 6 months. Of these patients, 3 had severe depression (HAMD score $>24$ ) and had been receiving inpatient care, and 2 patients had been taking a rest from their work for 1 month or more. Of the 3 patients having received inpatient care, 1 is now followed with fluvoxamine (an SSRI) at the maintenance dose $(25 \mathrm{mg})$ and 2 are now followed with paroxetine at a dose of $10 \mathrm{mg}$, and all are leading a favorable course. For the 2 patients who took a rest from their work, antidepressant therapy has been completed, and they are now followed with sleeping pills alone.

As of December 2010, all 5 of these patients had restored social activity. Three (age 55, 58, and 69 years) were working,

Table 2 Reduction of regional brain volume

\begin{tabular}{lllll}
\hline Location & MDD $(\mathbf{n}=\mathbf{7 I})$ & BD $(\mathbf{n}=\mathbf{I} \mathbf{I})$ & DC $(\mathbf{n}=\mathbf{6})$ & HC $(\mathbf{n}=\mathbf{3 3})$ \\
\hline Subgenual anterior cingulate cortex & $66(93.0 \%)$ & $6(54.5 \%)$ & $0(0 \%)$ & $5(\mathrm{I5.2 \% )}$ \\
Amygdala & $2 \mathrm{I}(29.6 \%)$ & $2(18.2 \%)$ & $0(0 \%)$ & $2(6.1 \%)$ \\
Hippocampus & $35(49.3 \%)$ & $4(36.4 \%)$ & $0(0 \%)$ & $2(6.1 \%)$ \\
Prefrontal cortex & $20(28.2 \%)$ & $2(18.2 \%)$ & $1(16.7 \%)$ & $1(3.0 \%)$ \\
\hline
\end{tabular}

Abbreviations: BD, bipolar disorder; DC, diagnostic change case; HC, healthy controls; MDD, major depression disorder. 
Table 3 Relationship between the characteristics and the reduction of SACC volume in MDD patients

\begin{tabular}{|c|c|c|c|c|c|}
\hline & \multicolumn{2}{|c|}{$\begin{array}{l}\text { Reduction } \\
\text { of } s A C C\end{array}$} & \multirow[t]{2}{*}{$\chi^{2}$} & \multirow[t]{2}{*}{ df } & \multirow[t]{2}{*}{$P$ value } \\
\hline & + & - & & & \\
\hline \multicolumn{6}{|l|}{ Sex } \\
\hline Male & 10 & 2 & 2.04 & 1 & 0.97 \\
\hline Female & 56 & 3 & & & \\
\hline \multicolumn{6}{|l|}{ Age, y } \\
\hline $54-63$ & 31 & 2 & 0.092 & 2 & 0.96 \\
\hline $64-73$ & 23 & 2 & & & \\
\hline $74-82$ & 12 & 1 & & & \\
\hline \multicolumn{6}{|l|}{ Site } \\
\hline Right & 61 & 10 & 2.24 & I & 0.13 \\
\hline Left & 54 & 17 & & & \\
\hline \multicolumn{6}{|l|}{ HAMD, point } \\
\hline-13 & 7 & 0 & 9.94 & 3 & 0.02 \\
\hline $14-18$ & 37 & 2 & & & \\
\hline $19-22$ & 15 & 0 & & & \\
\hline $23-$ & 7 & 3 & & & \\
\hline \multicolumn{6}{|l|}{ Duration of illness, $y$} \\
\hline $0.5-1$ & 9 & 0 & 1.69 & 3 & 0.64 \\
\hline $\mathrm{I}-3$ & 23 & I & & & \\
\hline $3-6$ & 17 & 2 & & & \\
\hline $6-$ & 17 & 2 & & & \\
\hline \multicolumn{6}{|c|}{ Duration of treatment, $y$} \\
\hline-0.5 & 6 & 0 & 3.62 & 4 & 0.46 \\
\hline $0.5-1$ & 15 & 0 & & & \\
\hline $\mathrm{I}-3$ & 19 & I & & & \\
\hline $3-6$ & 18 & 3 & & & \\
\hline $6-$ & 8 & 1 & & & \\
\hline \multicolumn{6}{|c|}{ Drug use (include combined use) } \\
\hline SSRI & 56 & 5 & 1.06 & 5 & 0.96 \\
\hline SNRI & 9 & 0 & & & \\
\hline NaSSA & 5 & 0 & & & \\
\hline Sulpiride & 3 & 0 & & & \\
\hline Tricycle anti-dep & 2 & 0 & & & \\
\hline Tetracycle ant-dep & I & 0 & & & \\
\hline
\end{tabular}

Abbreviations: anti-dep, antidepressant; HAMD, Hamilton's Rating Scale for Depression; MDD, major depression disorder; NaSSA, noradrenergic and specific serotonergic antidepressant; sACC, subgenual anterior cingulate cortex; SNRI, serotonin noradrenaline reuptake inhibitor; SSRI, selective serotonin reuptake inhibitor.

1 (age 66 years) was a housewife, and the other (age 79 years) was taking care of her husband at home as a housewife. All 5 of these patients retained cognitive function and were visiting the hospital once monthly while driving a car by themselves.

\section{Association between sACC volume reduction and modification of initial diagnosis of MDD}

Among the patients studied, there were 6 cases in which drug therapy with antidepressants after diagnosis of MDD in accordance with DSM-IV diagnostic criteria was ineffective and failed to result in remission. In all of these 6 cases,
MRI VSRAD revealed sACC volume reduction. The initial diagnosis was later modified in all these cases. The courses followed by these 6 cases can be summarized as:

\section{One case of sleep apnea syndrome complicated} by hypertension

Case 1: A 71-year-old male. Previously treated with SNRI for 3 years at another facility, but referred to our facility because of poor responses. At our facility, the diagnosis was modified, and CPAP therapy (continuous positive airway pressure for treatment of apnea) and blood control were performed, and resulted in alleviation of psychiatric symptoms and gradual reduction of antidepressant dose level to zero.

\section{Two cases markedly responding to pregabalin treatment}

Case 2: A 64-year-old male. Failed to respond to SNRI treatment, but responded markedly to pregabalin (a product marketed in Japan since July 2010) 1 week after the start of treatment. After its dose level was increased to $300 \mathrm{mg}$, all antidepressants were discontinued. The patient had been complaining of chronic lower back pain for the past 20 years. The lower back pain had not alleviated and the patient had been referred from an orthopedic clinic to our facility as a case of depression (the disease had not alleviated until recently).

Case 3: A 76-year-old male. Failed to respond to SNRI, but showed alleviation rapidly within 1 week after the start of pregabalin treatment. After its dose level was increased to $450 \mathrm{mg}$, all antidepressants were discontinued gradually. Pain due to cervical herniation had not alleviated after surgery. Patient was later diagnosed as having depression on the basis of sleeplessness and depressed mood, and treatment was started.

Both cases failed to satisfy the diagnostic criteria for fibrous myalgia and had been treated as cases of chronic pain complicated by depression.

\section{One case later developing Parkinson's disease}

Case 4: A 67-year-old male. Received SSRI therapy for depression but the disease did not alleviate. Later, the disease advanced into Parkinson's disease, which responded well to anti-Parkinsonian drugs.

\section{Two cases with economic issues and personal} relationship, and environmental problems

Case 5: A 55-year-old male. Case 6: A 64-year-old female. In both cases, the priority was to resolve economic issues and personal relationship and environmental problems. At early 
stages of treatment, antidepressants were additionally used, but they were ineffective and discontinued.

In these 6 cases, the finding of lack of sACC volume reduction revealed by MRI VSRAD was very useful, serving as an objective finding contributing to reviewing whether or not the initial diagnosis of MDD was appropriate. In other words, sACC volume reduction was found to serve as an important indicator for careful review of possible error in the initial diagnosis, instead of maintaining the diagnosis of MDD, in cases in which antidepressant therapy for the initially diagnosed MDD does not result in alleviation.

\section{Association between sACC volume reduction and diagnosis/treatment of bipolar disorder}

In all of the 11 cases with bipolar disorder diagnosed and treated in accordance with DSM-IV diagnostic criteria and subsequently followed with mood modulators, MRI was carried out 5 years or more after the start of treatment. The finding of sACC volume reduction was very useful in the management of these cases and, interestingly, it was consistent with the clinical course.

For the course and efficacy of treatment, these cases could be divided into the following 2 groups depending on the presence/absence of sACC volume reduction, and each group had different characteristics:

1. Group without sACC volume reduction (5/11 cases)

In all 5 of these cases, remission was maintained with mood modulators alone.

2. Group showing sACC volume reduction (6/11 cases)

In some of these 6 cases, the depressed phase repeated cycles of remission and relapse, making treatment difficult and requiring hospitalization during the depressed phase. Concomitant use of antidepressants was needed in this group.

\section{Examples of sACC volume reduction data in cases free of subjective symptoms}

Among the normal controls showing sACC volume reduction, 1 case (56-year-old female) later required sleeping pills. Detailed interview of this patient after acquisition of volume reduction data revealed difficulty in falling into sleep and experiencing fatigue. This case did not satisfy the criteria for diagnosis of MDD among the DSM-IV criteria and began to receive early intervention when HAMD score was 10 . At present, this woman is receiving sleeping pills only and can continue working.

The cases presented above were cases aged $>54$ years, the age group selected in accordance with the range of age included in MRI VSRAD. Using a similar method, we previously studied cases aged $<54$ years (40 cases) and obtained findings similar to those presented in this paper. This paper was confined to reporting cases aged $>54$ years analyzed with VSRAD criteria.

\section{Discussion}

This study analyzed morphological abnormalities of the brain (sACC volume reduction) associated with depression and evaluated the usefulness of VSRAD (a technique of VBM for such abnormalities) as an auxiliary diagnostic means. To our knowledge, this is the first study involving clear-cut analysis of the longitudinal and cross-sectional correlation of depression to MRI VSRAD. sACC volume reduction did not correlate with age ( $>54$ years), gender, disease severity, duration of illness, treatment period, type of drug used, or drug dose. sACC volume reduction remained to be seen even after remission (HAMD score $<7$ ). The evaluation of the association between SACC volume reduction and validity of treatments for depression and other diseases presenting with similar symptoms revealed that that if MRI VSRAD is utilized as an auxiliary diagnostic means of diagnosis of depression, it enables the prediction of prognosis in the following way.

1. In cases diagnosed as having MDD in accordance with DSM-IV diagnostic criteria and showing only sACC volume reduction, remission can be achieved by the use of antidepressants suitable for individual patients at appropriately adjusted doses, but continuation of antidepressant therapy at maintenance dose level will be needed after achievement of remission.

2. In cases diagnosed as having MDD in accordance with DSM-IV diagnostic criteria, showing no sACC volume reduction and responding well to antidepressants, the dose of antidepressants can be gradually reduced to zero. In these cases, close follow-up as to possible bipolar episodes will be needed.

3. In cases diagnosed as having MDD in accordance with DSM-IV diagnostic criteria, failing to show alleviation of symptoms and remission despite treatment and showing no sACC volume reduction, a finding of lack of sACC volume reduction is very useful when the initial diagnosis is reviewed fundamentally. The initial diagnosis is highly likely to be wrong in such cases, and review of the diagnosis is likely to enable prevention of erroneous diagnosis/treatment (repetition of usefulness dose increase or switching of antidepressants).

Numerous studies have reported abnormalities of the brain associated with depression. Neuroimaging studies of 
MDD, conducted to date, pertained to analysis of changes in grey matter volume of hippocampus, ${ }^{3-8}$ amygdala,,${ }^{9-11}$ striate body, ${ }^{12}$ prefrontal area, ${ }^{13,14}$ and anterior cingulate cortex, ${ }^{15-21}$ which are structures involved in the hypothamic-pituitaryadrenocortical system function, emotion/cognition, and adjustments. Many of these studies revealed grey matter volume reduction in these areas.

The present study involved analysis of the association of volume reduction in sACC, hippocampus, prefrontal area, and amygdala with MDD, but the sensitivity and true positive rate were low for volume reduction in hippocampus, prefrontal area, or amygdala when used as an indicator of MDD (Table 2). This result may be explained as follows. With VSRAD, areas with $Z$ score $>2$ (ie, areas with density differing by $2 \times \mathrm{SD}$ or more from the mean for normal database) are depicted as volume-reduced areas. The sensitivity was markedly high for volume reduction in $\mathrm{SACC}$, and this suggests that close attention should be paid to SACC when VSRAD is used.

The ACC, which contains SACC as a part, is a component of the cerebral cortex, and its anterior part forms the medial side of the frontal lobe (including Brodmann Area 24, 25, and 32). It is involved in cognitive function (attentiveness) and also in emotional responses as a part of the limbic system. In addition, ACC is involved also in dealing with pain and struggle. Among studies on sACC volume reduction, there is a report that $\mathrm{ACC}$ volume reduction was seen in all of 68 cases with MDD and 88 cases with MDD accompanied by anxiety disorder, and that sACC was noted in patients having developed depression at age $<18$ years. ${ }^{22}$

Among studies of brain function associated with sACC, using diagnostic imaging, there are studies during rest in patients with episodes of depression, demonstrating reduced cerebral blood flow and compromised metabolism in the anterior half of the cerebrum (particularly in the anterior part of cingulated cortex, subcallosal area, islet, orbital area, and mediodorsal-laterodorsal frontal area). ${ }^{23,24}$ Recently, many reports using functional MRI have been published, including a report showing that patients with depression have compromised function in the prefrontal area during implementation of a cognitive task, and hyperactivity of the limbic system (including the amygdala) during an emotional task. ${ }^{25}$ In studies on depression-associated brain function by diagnostic imaging, close attention has been paid to the brain areas showing changes after treatment and brain areas showing abnormal blood flow or metabolism after treatment. Generally, the reduction of cerebral blood flow and metabolism seen in the dorsolateral prefrontal area of untreated patients with depression is mood-dependent and tends to normalize after remission of symptoms, while the cerebral blood flow and metabolism in the medial prefrontal area tends to remain reduced even after alleviation of depression. This indicates occasional discrepancy between symptoms and brain function rated by diagnostic imaging. ${ }^{26}$

The findings from the patients studied suggest that if patients receiving treatment of depression showed sACC volume reduction, they probably required continued drug treatments even after remission of the symptoms of depression. The results suggest that VSRAD may be used as a means of following the course of symptoms of depression, with a focus set at the sACC, and judging the necessity of antidepressants after remission.

The next question pertains to the mechanism for reduction in the volume of sACC. It is known that excessive stress destroys the cellular function of responding to stress, inducing apoptosis of the cells. If nerve cells are exposed to stress, they lose viability, possibly resulting in compromised brain function and onset of various neurological diseases. Thus, cells are known to undergo not only extermination due to physical damages and intense disorders (eg, caused by toxins) but also death arising from lack of proliferation signals or various stimuli. These types of cellular death are called programmed death or apoptosis of cells. It is unknown whether the sACC volume reduction observed in the present study reflected apoptosis of nerve cells or a decrease in glial cell volume. According to a recent report, sACC volume reduction is most likely to reflect decrease in nerve cell volume and glial cell density. ${ }^{27}$ Further studies are needed on this issue.

For bipolar disorder, the present study has demonstrated that patients with depression complicated by bipolar disorder and showing sACC volume reduction responded to antidepressants, which resulted in remission. In patients with bipolar disorder alone (the uncomplicated group), sACC volume reduction was absent. In other words, these results suggest that patients with uncomplicated bipolar disorder respond to treatment with mood modulators alone, while patients with depression complicated by bipolar disorder are expected to respond to combined mood modulator plus antidepressant therapy. This retrospective finding suggests that MRI VARAD may be useful when judging the necessity of combined use of antidepressants or continued combined use of antidepressants during the depressed phase of patients receiving treatment on the basis of the diagnosis of bipolar disorder. After our detection of the presence of an ACC volume reduction group among patients with bipolar disorder, 
a paradigm shift (treatment strategy based on awareness of the presence of bipolar disorder plus MDD cases) was useful in our clinical practice. MRI VSRAD will be also useful when treating cases diagnosed as having bipolar disorder if these cases show sACC volume reduction, in view of the possibility that these cases are actually in a drug-induced manic phase of depression following treatment with inappropriate antidepressants and they should be managed as cases of depression. Our conclusion at present is that the volume reduced area of sACC probably responds to antidepressants. Depression differs in disease characteristics from bipolar disease and it involves longitudinal changes of brain morphology. Brain sites possibly involved in bipolar disorder are diverse. ${ }^{28}$ For diagnostic imaging, there is a report that tractograms, yielded from tracing of the fibers of anterior thalamic radiation on diffusion tensor images, revealed reduced cross-sectional area of the anterior thalamic radiation, suggesting disturbed white matter between thalamus and anterior lobe at the bilateral anterior thalamic radiation as well as association of bilateral disturbance in nerve development with the pathogenesis of bipolar disorder. ${ }^{29}$ Further studies on this topic are also desirable.

When treatments are selected and the prognosis is predicted, it is significant to define depression as "a condition involving sACC volume reduction and presenting with psychiatric symptoms". That is, the volume reduction in this area serves as an indicator of vulnerability to stress. When dealing with patients showing sACC volume reduction and presenting with related psychiatric symptoms (sleeplessness, depressed mood, fatigue, and pain), it is advisable to consider the necessity of treatment with antidepressants on the basis of assessment of psychiatric symptoms through detailed interview. Instead of relying on the conventional ways of diagnosis and treatment based solely on the features of depression and bipolar disorder, it is desirable to adopt a basic stance by which some particular areas of the brain (eg, sACC) are assessed for anomaly (volume reduction) and treatments (antidepressants) to make up for the lost function are considered. If the view that antidepressants are effective in cases showing sACC volume reduction is endorsed, it will be very useful during clinical practice.

\section{Open issues}

The results from the present study do not allow us to readily conclude that the absence of SACC volume reduction suggests antidepressants to be unnecessary. According to a report presented at the 28th Meeting of the Japanese Society for Magnetic Resonance in Medicine 2010, calculation of the hippocampal $Z$ score with MRI VSRAD has been used as an auxiliary means of diagnosing dementia, despite concern that the spread of this technique could result in excessive reliance on numerical data (not combined with the original images) in diagnosis of dementia. This means that even when the usefulness of MRI as a means of diagnostic imaging is widely accepted, diagnosis of depression solely on the basis of MRI findings should be avoided. Clinicians need to deal with their patients in a modest and calm manner.

In future, more studies are awaited on the relationship between genes and SACC volume reduction:

1. Regarding the validity of checking for sACC volume reduction in the diagnosis of depression in individuals aged $>54$ years, it is necessary to carry out large-scale studies at other medical facilities and research facilities. Clinical studies on a global scale are desirable.

2. If attention is paid to $\mathrm{SACC}$ volume reduction at the time of diagnosis based on MRI during thorough brain checks, for example, it may be possible to identify high-risk individuals from the viewpoint of prevention of depression. Linkage between the psychiatric department and the brain diagnostic imaging department, among others, needs to be considered.

3. If a computer program enabling application of reference normal images to younger generations (20s through 40s) is developed, it will also serve as an objective indicator of depression among young individuals. Nations with a high prevalence of depression among their young generations will lose vitality and may decline. Global utilization of simply and immediately applicable MRI VARAD will enable appropriate diagnosis and treatment of depression, covering all generations.

It is also important to analyze the phenotypes at various levels (such as brain nerve circuits, brain function, brain structure, molecule/gene expression, gene polymorphism) and to attempt further classification of depression (a disease consisting of diverse conditions) and further evaluation of its features.

\section{Conclusion}

The present study has analyzed the potential of VSRAD as an auxiliary means of diagnosing depression in clinical cases. By showing that sACC volume reduction was seen frequently among patients receiving treatment of MDD, the authors have demonstrated the usefulness of SACC volume reduction as an objective auxiliary means of diagnosing MDD and that depression differs in terms of disease characteristics from bipolar disease, which has the potential to involve longitudinal changes of brain morphology. 
At present, studies with a molecular biology approach are being performed, involving measurement of genes and biomaterials associated with depression. Numerous reports of case of depression diagnosed using near-infrared spectroscopy of brain function have been published. However, this kind of device has not become widespread in Japan. Even though positron emission tomography (PET), which visualizes glucose metabolism with radioisotopes, has been shown to be useful in evaluation of brain function and blood flow, the number of facilities equipped with devices for nuclear medicine is smaller than that of facilities equipped with MRI devices, and the cost of PET is higher than that of MRI. The number of MRI devices available for use is very large in Japan compared with many other countries, with more than 6000 MRI devices in use in Japan. VSRAD can be characterized by its capability of visual and objective judgment as to the site of change and the extent of change even by physicians not well experienced with diagnostic imaging. It thus yields objective data about the condition of individual patients which can be shared between the physician and the patient or among physicians.

(Note: An anonymous reviewer has pointed out that Fabienne et al (2011) has demonstrated that conditions of corpus callosum are directly related to suicidality in older individuals. Thus far, we have not been able to obtain the paper because it has not been published yet. A similar argument is also made by Ballmaier et al..$^{30}$ VSRAD is a software program that measures conditions of grey mater, thus examinations of corpus callosum are beyond the scope of this paper. Nevertheless, a software program capable of measuring conditions of white matter is currently under development, and further research is awaited).

The number of individuals who committed suicide in Japan has exceeded 30,000 per year every year since 1998 , and depression is considered to be involved in $60 \%$ of all suicides. If more MRI devices in Japan are used to detect sACC volume reduction (an indicator of vulnerability to stress), MRI will be very effective in preventing suicide. This is because the combination of MRI and VSRAD is useful as an auxiliary means of diagnosis of depression, enabling automated detection of sACC volume reduction even by physicians of nonpsychiatric specialties, in addition to psychiatrists having received clinical training on depression, thus facilitating early detection of depression across the country. This will lead to prevention of suicide. Patients showing sACC volume reduction have the potential to respond to early intervention if they are observed as the risk factor group (vulnerable to stress) even when they are currently free of psychiatric symptoms. If MRI VSRAD can be introduced as a part of thorough brain checks to establish a system for detection of sACC volume reduction, it will facilitate early detection and treatment of depression. To conclude, we hope that this study will trigger dissemination of the awareness that the finding of SACC volume reduction as a sign of depression is useful as an auxiliary means for prevention of suicide and that data endorsing this view are collected on a global scale.

\section{Acknowledgments}

The authors are grateful to the editor and the three anonymous reviewers for their constructive comments and suggestions on this study, which we found very valuable. We are also indebted to Medical English Service for their excellent work in translating the Japanese manuscript into English. Special thanks go to Dr Yasuyo Hijikata (Toyodo Hijikata Clinic) and Ms Takako Kuniyoshi (Nanto Clinic) for their warm encouragement and support.

\section{Disclosure}

The authors declare no conflicts of interest.

\section{References}

1. Pezawas L, Meyer-Lindenberg A, Drabant EM, et al. 5-HTTLPR polymorphism impacts human cingulate-amygdala interactions: a genetic susceptibility mechanism for depression. Nat Neurosci. 2005;8(6): 828-834.

2. Hirata Y, Matsuda H, Nemoto K, et al. Voxel-based morphometry to discriminate early Alzheimer's disease from controls. Neurosci Lett. 2005;382(3):269-274.

3. Frodl T, Schaub A, Banac S, et al. Reduced hippocampal volume correlates with executive dysfunctining in major depression. J Psychiatry Neurosci. 2006;31(5):316-323.

4. Lange C, Irle E. Enlarged amygdala volume and reduced hippocampal volume in young women with major depression. Psychol Med. 2004;34(6):1059-1064.

5. MervaaIa E, Föhr J, Könönen M, et al. Quatitative MRI of the hippocampus and amygdala in severe depression. PsychoI Med. 2000;30(1): 117-125.

6. Yucel K, McKinnon MC, Chahal R, et al. Anterior cingulate volumes in never-treated patients with major depressive disorder. Neuropsychopharmacology. 2008;33(13):3157-3163.

7. Sheline YI, Gado MH, Kraemer HC. Untreated depression and hippocampal volume loss. Am J Psychiatry. 2003;160(8):1516-1518.

8. Campbell S, MacQueen G. An update on regional brain volume differences associated with mood disorders. Curr Opin Psychiatry. 2006; 19(1):25-33.

9. Yoshikawa E, Matsuoka Y, Yamasue H, et al. Prefrontal cortex and amygdala volume in first minor or major depressive episode after cancer diagnosis. Biol Psychiatry. 2006;59(8):707-712.

10. Wagner G, Koch K, Schachtzabel C, et al. Enhanced rostral anterior cingulate cortex activation during cognitive control is related to orbitofrontal volume reduction in unipolar depression. J Psychiatry Neurosci. 2008;33(3):199-208.

11. Sheline YI, Gado MH, Price JL. Amygdala core nuclei volumes are decreased in recurrent major depression. Neuroreport. 1998;9(9): 2023-2028. 
12. Hickie IB, Naismith SL, Ward PB, et al. Serotonin transporter gene status predicts caudate nucleus but not amygdala or hippocampal volumes in older persons with major depression. J Affect Disord. 2007; 98(1-2):137-142.

13. Taki Y, Kinomura S, Awata S, et al. Male elderly subthreshold depression patients have smaller volume of medial part of prefrontal cortex and precentral gyrus compared with age-matched normal subjects: a voxel-based morphometry. J Affect Disord. 2005;88(3):313-320.

14. Bremner JD, Vythilingam M, Vermetten E, et al. Reduced volume of orbitofrontal cortex in major depression. Biol Psychiatry. 2002; 51(4):273-279.

15. Drevets WC, Price JL, Simpson JR Jr, et al. Subgenual prefrontal cortex abnormalities in mood disorders. Nature. 1997;386(6627):824-827.

16. Frodl T, Jäger M, Born C, et al. Anterior cingulate cortex does not differ between patients with major depression and healthy controls, but relatively large anterior cingulate cortex predicts a good clinical course. Psychiatry Res. 2008;163(1):76-83.

17. Yuan Y, Zhu W, Zhang Z, et al. Regional gray matter changes are associated with cognitive deficits in remitted geriatric depression: an optimized voxel-based morphometry study. Biol Psychiatry. 2008; 64(6):541-544.

18. Hastings RS, Parsey RV, Oquendo MA, et al. Volumetric analysis of the prefrontal cortex, amygdala, and hippocampus in major depression. Neuropsychopharmacology. 2004;29(5):952-959.

19. Tang Y, Wang F, Xie G, et al. Reduced ventral anterior cingulate and amygdala volumes in medication-naive females with major depressive disorder: a voxel-based morphometric magnetic resonance imaging study. Psychiatly Res. 2007;156(1):83-86.

20. Koolschijn PC, van Haren NE, Lensve1t-Mulders GJ, et al. Brain volume abnormalities in major depressive disorder: a meta-analysis of magnetic resonance imaging studies. Hum Brain Mapp. 2009;30(11): 3719-3735.
21. Drevets WC, Savitz J, Trimble M. The subgenual anterior cingulate cortex in mood disorders. CNS Spectr. 2008;13(8):663-681.

22. van Tol MJ, van der Wee NJ, van den Heuvel OA, et al. Regional brain volume in depression and anxiety disorders. Arch Gen Psychiatry. 2010; 67(10):1002-1011.

23. Galynker II, Cai J, Ongseng F, et al. Hypofrontality and negative symptoms in major depressive disorder. J Nucl Med. 1998;39(4):608-612.

24. Hanada K, Hosono M, Kudo T, et al. Regional cerebral blood flow in the assessment of major depression and Alzheimer's disease in the early elderly. Nucl Med Commun. 2006;27(6):535-541.

25. Siegle GJ, Thompson W, Carter CS, et al. Increased amygdala and decreased dorsolateral prefrontal BOLD responses in unipolar depression: related and independent features. Biol Psychiatry. 2007; 61(2):198-209.

26. Ishizaki J, Yamamoto H, Takahashi T, et al. Changes in regional cerebral blood flow following antidepressant treatment in late-life depression. Int J Geriatr Psychiatry. 2008;23(8):805-811.

27. Cotter D, Mackay D, Landau S, et al. Reduced glial cell density and neuronal size in the anterior cingulate cortex in major depressive disorder. Arch Gen Psychiatry. 2001;58(6):545-553.

28. Berton O, Nestler EJ. New approaches to antidepressant drug discovery: beyond monoamines. Nat Rev Neurosci. 2006;7(2):137-151.

29. Ikeda A, Kito S, Jung J, et al. Decreased cross-sectional area of the anterior thalamic peduncle in bipolar disorder: a fiber tracking study. J Intl Soc Life Info Sci. 2010;28(1):14-20.

30. Ballmaier M, Kumar A, Elderkin-Thompson V, et al. Mapping callosal morphology in early- and late-onset elderly depression: an index of distinct changes in cortical connectivity. Neuropsychopharmacology. 2008;33(7):1528-1536.
International Journal of General Medicine

\section{Publish your work in this journal}

The International Journal of General Medicine is an international, peer-reviewed open-access journal that focuses on general and internal medicine, pathogenesis, epidemiology, diagnosis, monitoring and treatment protocols. The journal is characterized by the rapid reporting of reviews, original research and clinical studies across all disease areas.

\section{Dovepress}

A key focus is the elucidation of disease processes and management protocols resulting in improved outcomes for the patient.The manuscript management system is completely online and includes a very quick and fair peer-review system. Visit http://www.dovepress.com/ testimonials.php to read real quotes from published authors. 